Classification

Physics Abstracts

$79.60-06.70 \mathrm{D}-07.80$

\title{
Electron Compton scattering in a symmetric two-beam scattering geometry
}

\author{
Peter Jonas and Peter Schattschneider $\left(^{*}\right)$ \\ Institut für Angewandte und Technische Physik, Technische Universität Wien, Wiedner Hauptstraße \\ 8-10, A-1040 Wien
}

(Received April 9; accepted May 14, 1993)

\begin{abstract}
The aim of this work is to present an experimental procedure to measure directional Compton profiles by means of electron energy-loss spectroscopy in the transmission electron microscope. In order to obtain unique profiles with a well defined orientation in momentum space a symmetric two-beam scattering geometry taking advantage of crystal symmetry was developped. Measurements on single crystal silicon are discussed in comparison to results of other Compton scattering techniques and theoretical calculations.
\end{abstract}

\section{Introduction.}

The momentum density distribution of charge carriers in solids can be probed in a transmission electron microscope equipped with an electron energy-loss spectrometer by means of a technique called electron Compton scattering from solids (ECOSS) [22, 23, 10].

The central quantity in Compton scattering, the Compton profile

$$
J\left(p_{z}\right)=\int d^{2} p_{x y} \rho\left(p_{x y}, p_{z}\right)
$$

is a projection of the 3-dimensional momentum density $\rho(\mathbf{p})$ onto the direction of the scattering vector q. For single scattering and under the validity of the impulse approximation (IA) [5], i.e for large momentum transfer, the double differential scattering cross section for inelastic electron scattering relates to the Compton profile as

$$
\frac{\partial^{2} \sigma}{\partial E \partial \Omega}=\left[\frac{2 m_{e} e^{2}}{(\hbar q)^{2}}\right]^{2} \frac{k_{b}}{k_{a}} \frac{m_{e}}{4 \pi^{2} q} \cdot J\left(p_{z}\right)
$$

where $q$ is the modulus of the scattering vector.

$\left({ }^{*}\right)$ also at: Ecole Centrale Paris, laboratoire de mecanique, F-92295 Chatenay-Malabry, France. 
Recent work has shown the feasibilty of our combined Jeol 200CX-TEM, GATAN 666-PEELSsystem for experimental Compton work $[16,8,17]$. The effective momentum resolution, depedend on the angle of convergence of the primary electron beam incident on the specimen, spherical and chromatic aberration of the post specimen lenses, the diameter of the spectrometer and finally by the energy resolution, which can be achieved at present with our system is $\delta p_{z}=0.135$ atomic units ${ }^{1}$ [9]. By means of Fourier-techniques based simulations the impact of multiple scattering upon the Compton profiles have been shown; deconvolution routines and an approach to the elastic background problem were developped $[21,9,10]$.

The scope of this work is to discuss on the example of single crystal silicon, (i) a special kind of scattering geometry taking advantage of crystal symmetry which enables us to measure unique profiles with a well defined direction in momentum space and (ii) some results of electron scattering in comparison with theoretical Compton profiles and the outcome of photon Compton measurements.

\section{Specimens.}

Silicon was chosen to serve as test material since it is well documented in literature, easy to prepare for the microscope and quite inert to oxidation. A number of experiments with aluminum showed that materials which are easy contaminated by oxygen are not suited for electron Compton measurements; results of aluminum will therefore not be presented since they bear no scientific information except that oxidation is a huge problem related to this kind of transmission scattering technique. The reason is that one ought to use specimens as thin as possible to reduce multiple scattering and therefore an additional oxygen-layer strongly alters the outcome by covering the real signal to be measured.

Single crystal samples have been available in three orientations [100], [110] and [111]. Disks of $3 \mathrm{~mm}$ diameter have been cut out of the respective wafer by means of an ultrasonic drill and were mechanical thinned on a fast rotating cast iron disk to obtain a flat parallel-sided sample of thickness around $100 \mu \mathrm{m}$. A dimple grinder was used for grinding circular dimples on one side down to a remaining thickness in the middle of the depression of $30 \mu \mathrm{m}$. Finally $\mathrm{Ar}^{+}$-ion milling was used to get specimen areas of reasonable thickness for the purpose of EELS, i.e. $d \leq 150 \mathrm{~nm}$ on the edge of the narrow hole which occurs due to the ion bombardement.

The thinning process showed reasonable results although a compromise had to be made between the requirements concerning the thickness of the specimen which had to be as thin as possible and the fact that the crystal tended to bend the thinner it was. Due to the fact that the samples available were bent it was necesarry to focus the electron beam on areas not larger than 3-4 $\mu \mathrm{m}$ in diameter in order to assure that the part of the crystal illuminated had a well defined orientation.

\section{Symmetric two-beam case.}

Using single crystal specimens to measure directional Compton profiles, care has to be taken in choosing a proper scattering geometry. The reason is that strong contributions from BraggCompton channel coupling may overlap the directly scattered Compton intensity and alter the result dramatically. Each Bragg beam, having been diffracted through a large angle relative to the incident beam, may undergo Compton scattering into the direction of the spectrometer aperture. A set of various Compton profiles with different maximum, width and direction in momentum space is superimposed on the single scattering profile.

\footnotetext{
$\left({ }^{1}\right)$ The unit of momentum $p_{z}$ will be given in 'atomic units' as usual in Compton scattering literature, 1 a.u. $\equiv \hbar a_{0}^{-1}$,
} $\hbar \equiv 1$. 


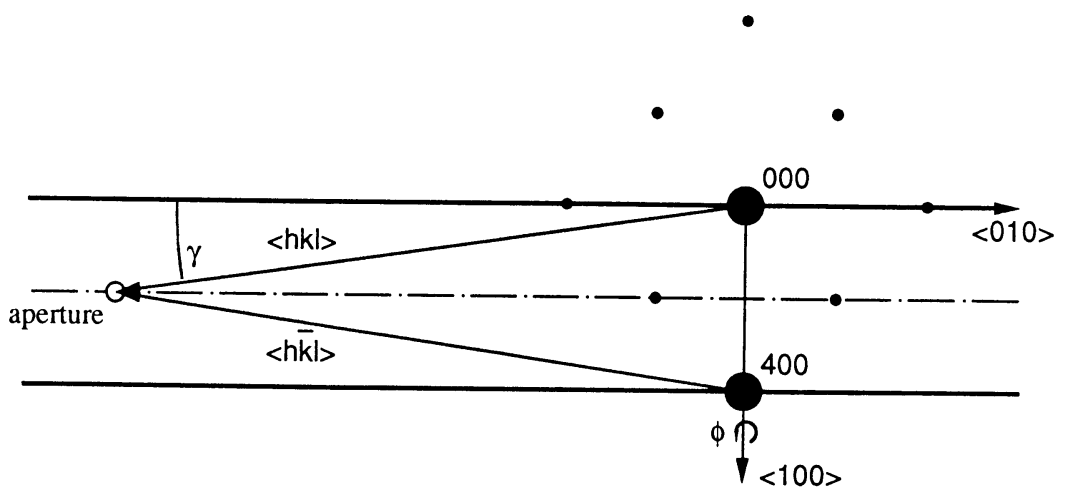

Fig.1. - Symmetric 2 beam scattering geometry.

Coherent effects (superposition of amplitudes instead of intensities from different directions) arising from the fact that the primary electron splits into a sum of Bloch waves within the crystal have been shown to be neglicible [10].

Williams calculated the incoherent multiple scattering contributions due to Bragg scattering in the high energy and high momentum transfer regime using a multi-slice approach that allows for dynamical scattering and treating each Bragg beam as a source of Compton scattering [24]. The Bragg-Compton channel coupling contributions alter the shape of the single Compton profile on the low energy-loss side as well as on the high energy-loss side thus it is impossible to get a useful result for a single crystal in a zone axis orientation. So the goal is to reduce the amount of Braggdiffracted beams which may contribute to the measurement and find a scattering geometry where Compton profiles of one scattering angle and equivalent directions in momentum space can be measured.

The demand for a reduction of the number of Bragg-diffracted beams can be achieved by tilting the specimen away from the zone-axis into a two-beam case. Placing the spectrometer aperture exactly on the axis of symmetry we now measure two Compton profiles of the same scattering angle ${ }^{2}$ and if the crystal obeys certain symmetry relations it is possible that these two Compton profiles are of equal direction in momentum space (see Fig. 1). We will discuss this point in the following particullary for the case of silicon.

We use the fact that the momentum density distribution $\rho(\mathbf{p})$ must be invariant to all operations of the point group of the crystal lattice in addition to having inversion symmetry $\rho(\mathbf{p})=\rho(-\mathbf{p})$ [14]. Then the Compton profile as a projection of $\rho(\mathbf{p})$ of course must follow the same symmetry. Silicon is of diamond type structure (space group 227), i.e. we have a face centred cubic lattice with a basis of two atoms located at $\left(\frac{1}{8}, \frac{1}{8}, \frac{1}{8}\right)$ and $\left(-\frac{1}{8},-\frac{1}{8},-\frac{1}{8}\right)$ where the origin has been chosen such that the crystal features an inversion centre. The point group of this structure is denoted $m 3 m$ in the international (Hermann-Maugin) notation [6]. The elements of this point group are

$$
\begin{array}{llllllllllllllllllllllll}
E & 2 & 3_{j}^{+} & 3_{j}^{-} & 2_{p} & 4_{i}^{+} & 4_{i}^{-} & I & \overline{2}_{i} & \overline{3}_{j}^{+} & \overline{3}_{j}^{-} & \overline{2}_{p} & \overline{4}_{i}^{+} & \overline{4}_{i}^{-},
\end{array}
$$

with the following point operations
$E \quad$ Identity operation
$n_{k}^{+} \quad$ Rotation for an angle $2 \pi / n$ counter-clockwise
$n_{k}^{-} \quad$ Rotation for an angle $2 \pi / n$ clockwise, around the axis $\mathbf{e}_{k}$

$\left({ }^{2}\right)$ We will therefore refer to this kind of geometry as the 'symmetric' 2-beam case hereafter. 
I Inversion

$\bar{n}_{k}^{+} \quad$ Rotation for an angle $2 \pi / n$ counter-clockwise, followed by inversion

$\bar{n}_{k}^{-} \quad$ Rotation for an angle $2 \pi / n$ clockwise, around the axis $\mathbf{e}_{k}$, followed by inversion.

The axis $\mathbf{e}_{k}$ are given in a cartesian coordinate system

$$
\mathbf{e}_{p}\left\{\begin{array} { l } 
{ \mathbf { e } _ { a } = ( 1 , 1 , 0 ) / \sqrt { 2 } } \\
{ \mathbf { e } _ { b } = ( - 1 , 1 , 0 ) / \sqrt { 2 } } \\
{ \mathbf { e } _ { c } = ( 1 , 0 , 1 ) / \sqrt { 2 } } \\
{ \mathbf { e } _ { d } = ( 0 , 1 , 1 ) / \sqrt { 2 } } \\
{ \mathbf { e } _ { e } = ( - 1 , 0 , 1 ) / \sqrt { 2 } } \\
{ \mathbf { e } _ { f } = ( 0 , - 1 , 1 ) / \sqrt { 2 } }
\end{array} \quad \mathbf { e } _ { j } \quad \left\{\begin{array} { l } 
{ \mathbf { e } _ { 1 } = ( 1 , 1 , 1 ) / \sqrt { 3 } } \\
{ \mathbf { e } _ { 2 } = ( - 1 , - 1 , 1 ) / \sqrt { 3 } } \\
{ \mathbf { e } _ { 3 } = ( 1 , - 1 , - 1 ) / \sqrt { 3 } } \\
{ \mathbf { e } _ { 4 } = ( - 1 , 1 , - 1 ) / \sqrt { 3 } }
\end{array} \quad \mathbf { e } _ { i } \left\{\begin{array}{l}
\mathbf{e}_{x}=(1,0,0) \\
\mathbf{e}_{y}=(0,1,0) \\
\mathbf{e}_{z}=(0,0,1)
\end{array}\right.\right.\right.
$$

Requesting to obtain Compton profiles of equivalent directions in momentum space in the symmetric two-beam case means that $J(h k l) \equiv J(\bar{h} k l)$ and equivalent relations for $k$ and $l$. This demands the existence of a mirror plane parallel to the $\mathbf{e}_{i}$ or, because a reflection is equal to a rotation for $\pi$ followed by an inversion, the existence of the symmetry operation $\overline{2}_{i}$, which is an element of the point group $m 3 m$. Since $\rho(\mathbf{p})=\rho(-\mathbf{p})$ the condition that $J(h k l) \equiv J(\bar{h} k l)$ is fulfilled for every crystal system featuring a point group with the element $2_{i}$.

Using the two-beam technique has its consequences upon the directions $p_{z}$ availiable for the measurement. We will explain that in more detail for a (400)-two beam case adjacent to the [001]zone axis. A tilt around an axis $\mathbf{e}=\left(e_{x}, e_{y}, e_{z}\right)$ for an angle $\phi$ transforming the vector $\mathbf{r}$ into $\mathbf{r}^{\prime}$ is described by a matrix such

$$
\mathbf{r}^{\prime}=\hat{\alpha} \mathbf{e}(\phi) \mathbf{r}
$$

with

$\hat{\alpha} \mathbf{e}(\phi)=\cos \phi\left(\begin{array}{ccc}1 & 0 & 0 \\ 0 & 1 & 0 \\ 0 & 0 & 1\end{array}\right)+(1-\cos \phi)\left(\begin{array}{ccc}e_{x} e_{x} & e_{x} e_{y} & e_{x} e_{z} \\ e_{x} e_{y} & e_{y} e_{y} & e_{y} e_{z} \\ e_{x} e_{z} & e_{y} e_{z} & e_{z} e_{z}\end{array}\right)+\sin \phi\left(\begin{array}{ccc}0 & -e_{z} & e_{y} \\ e_{z} & 0 & -e_{x} \\ -e_{y} & e_{x} & 0\end{array}\right)$

e must be of unit lenght $|\mathbf{e}|=e_{x} e_{x}+e_{y} e_{y}+e_{z} e_{z}=1$.

Placing the spectrometer aperture on the symmetry axis between the two Bragg reflections, the direction of the momentum component, if the crystal is the pole-orientation, would be $p_{z} \|$ $\langle 1,8,0\rangle, \gamma$ degrees away from the direction of the symmetry axis. $\gamma$ is given by $\sin \gamma=k_{G} / 2 q$, for a typical scattering angle $q=15.2 \AA^{-1}$ and $k_{4,0,0}=4.6 \AA^{-1}$ we get $\gamma=8.7^{\circ}$. Tilting the crystal about the $\langle 1,0,0\rangle$ direction out of the $[0,0,1]$-zone axis diminishes the intensity of the Bragg spots parallel to the $(4,0,0)$-systematic row to almost zero. The rotation angle $\phi$ necessary to achieve a proper two-beam condition depends on the specific geometry of the spot pattern and the selected Bragg reflection, for the $(4,0,0)$-reflection it ranges around $4^{\circ}$. For the momentum component $p_{z}$ actually measured we obtain by inserting a value of $\phi=4^{\circ}$ into (3) with $\vec{e}=(0,0,1), p_{z} \|\langle\overline{3}, 19,2\rangle$.

The scattering vector does not coincide with a high symmetry direction any more. The deviation angle $\alpha(h s \mid 2 b)$ from a high symmetry direction is given with $\phi$ and $\gamma$

$$
\cos \alpha=\cos \phi \cdot \cos \gamma
$$

The experimentally observed tilt angles $\phi$ to achieve more or less ideal 2-beam conditions are listed in table I together with the resulting directions in momentum space which are accesible within the respective symmetric two beam scattering geometry related to those values of momentum transfer $q$ used in the respective measurement as presented in section 7 . 
Table I. - High symmetry and accesible directions for the several two beam cases.

\begin{tabular}{|c|ccccc|}
\hline zone axis & $\begin{array}{c}\text { Bragg } \\
\text { reflection }\end{array}$ & $\begin{array}{c}\text { high symmetry } \\
\text { direction (hs) }\end{array}$ & $\begin{array}{c}\text { 2-beam } \\
\text { direction }(2 \mathrm{~b})\end{array}$ & $\begin{array}{c}\text { deviation } \\
\text { angle } \\
\text { and }\end{array}$ & $\begin{array}{c}\text { tilt } \\
\text { angle }\end{array}$ \\
\hline$[001]$ & $(2,2,0)$ & $\langle h k l\rangle$ & $\langle h k l\rangle$ & $\alpha(h s \mid 2 b)$ & $\phi$ \\
& $(4,0,0)$ & $\langle 0,1,0\rangle$ & $\langle\overline{3}, 19,2\rangle$ & $10.7^{\circ}$ & $4^{\circ}$ \\
\hline$[111]$ & $(2, \overline{2}, 0)$ & $\langle 1,1, \overline{2}\rangle$ & $\langle 12,16, \overline{2} 2\rangle$ & $8.7^{\circ}$ & $5^{\circ}$ \\
& $(\overline{2}, \overline{4}, 2)$ & $\langle 1,0, \overline{1}\rangle$ & $\langle\overline{4}, 19,22\rangle$ & $9.0^{\circ}$ & $5^{\circ}$ \\
\hline$[011]$ & $(4, \overline{2}, 2)$ & $\langle 1,1, \overline{1}\rangle$ & $\langle 4,9, \overline{5}\rangle$ & $19.8^{\circ}$ & $11^{\circ}$ \\
\hline
\end{tabular}

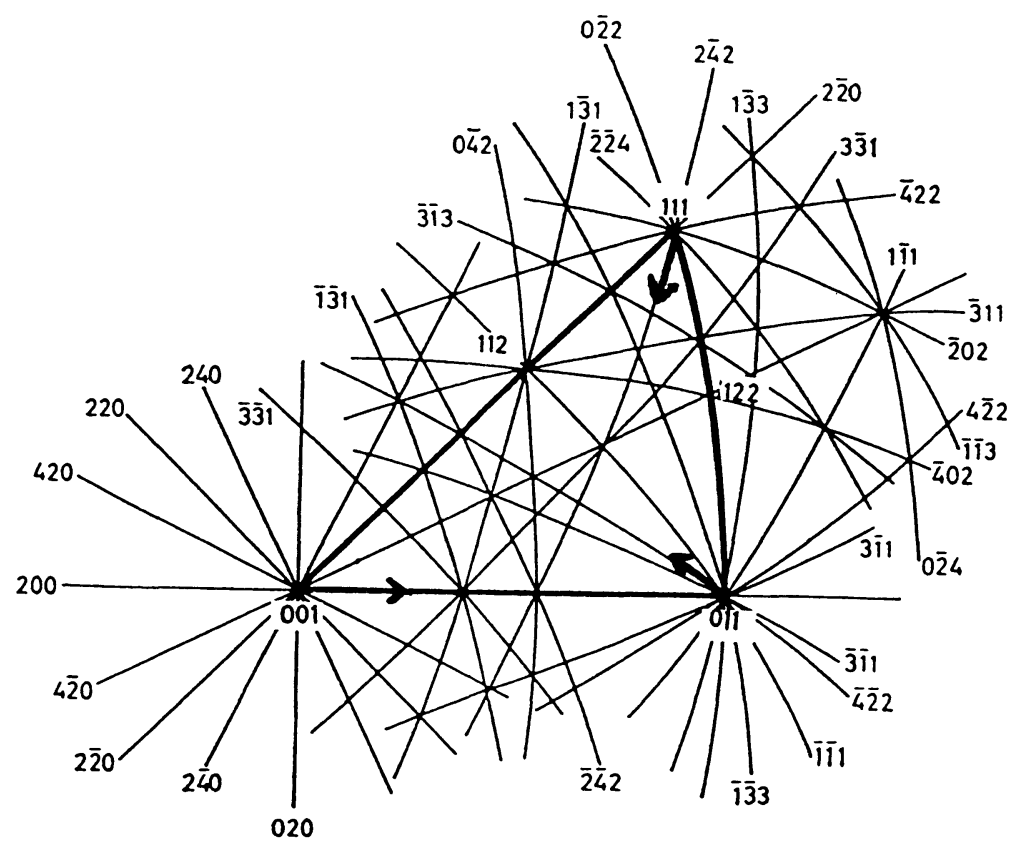

Fig.2. - Traces of the crystal planes plotted as a stereographic projection around the spherical triangle build up of the [001], [011] and [111] zone axis for a face-centred cubic structure. The arrows indicate the tilt direction for the several 2-beam cases. Graphic from [7].

Another consequence of the fact that we are not totaly free in choosing the direction in momentum space is that we might loose information due to the high symmetry of the crystal; we will discuss this point for the high indexed Bragg reflections in table I in the following. The geometrical conditions for a fcc structure are illustrated in figure 2. Due to the high symmetry of the diamond structure all anisotropies in momentum density properties are confined within the triangle [001] - [011] - [111]. Tilting the crystal away from the high symmetry directions means that the effective measured direction in momentum space moves along the boundaries of the triangle or in general it moves into the triangle. Thus the angles between the several directions accesible become smaller or to put it physical the anisotropies that we measure, diminish. 
Table II. - Angles between the high symmetry directions, respective between the 2-beam directions.

$$
\begin{array}{|l|c|}
\hline \alpha(\langle 1,0,0\rangle \mid\langle 1,1,0\rangle)=45^{\circ} & \alpha^{\prime}(\langle 19,3,2\rangle \mid\langle 22,19,4\rangle)=31.7^{\circ} \\
\beta(\langle 1,0,0\rangle \mid\langle 1,1,1\rangle)=54^{\circ} & \beta^{\prime}(\langle 19,3,2\rangle \mid\langle 9,5,4\rangle)=24.7^{\circ} \\
\gamma(\langle 1,1,0\rangle \mid\langle 1,1,1\rangle)=35^{\circ} & \gamma^{\prime}(\langle 22,19,4\rangle \mid\langle 9,5,4\rangle)=17.6^{\circ} \\
\hline
\end{array}
$$

Table III. - Relative intensities of the several Bragg beams within the sytematic row $-\mathbf{g}, 0, \mathbf{g}, 2 \mathbf{g}$ with $\mathrm{g}$ in the exact Bragg-condition $(w=0)$, averaged over specimen thickness $d=50 \mathrm{~nm}$, resp. $d=150$ $n m$.

\begin{tabular}{|c|cccc|}
\hline systematic row & $(0, \overline{2}, \overline{2})$ & $(0,0,0)$ & $(0,2,2)$ & $(0,4,4)$ \\
\hline$d=50 \mathrm{~nm}$ & 0.016 & 1 & 1.09 & 0.01 \\
$d=150 \mathrm{~nm}$ & 0.015 & 1 & 1.08 & 0.011 \\
\hline systematic row & $(0,0, \overline{4})$ & $(0,0,0)$ & $(0,0,4)$ & $(0,0,8)$ \\
\hline$d=50 \mathrm{~nm}$ & $1.3 \times 10^{-3}$ & 1 & 0.64 & $7.2 \times 10^{-4}$ \\
$d=150 \mathrm{~nm}$ & $1.5 \times 10^{-3}$ & 1 & 1.03 & $1.0 \times 10^{-3}$ \\
\hline systematic row & $(2, \overline{2}, \overline{4})$ & $(0,0,0)$ & $(\overline{2}, 2,4)$ & $(\overline{4}, 4,8)$ \\
\hline$d=50 \mathrm{~nm}$ & $2.9 \times 10^{-4}$ & 1 & 0.50 & $1.5 \times 10^{-4}$ \\
$d=150 \mathrm{~nm}$ & $3.7 \times 10^{-4}$ & 1 & 1.24 & $2.8 \times 10^{-4}$ \\
\hline
\end{tabular}

Additionally, the tilt angle $\phi$ to achieve the $(4, \overline{2}, 2)$-case out of the [011]-zone axis is 2 -fold larger then the tilt angles in the vicinity of the other pole orientations, which may be explaind by the higher density of Bragg reflections in the [011] orientation compared to [001] and [111], thus one has to tilt more strongly out of the zone axis to achieve a proper 2-beam condition. The effective angles between the three directions which can be measured with the three high indexed 2-beam cases from table I are listed in table II.

The question arises whether the side reflections of the two Bragg spots are weak enough to be neglicible. For this purpose the three different systematic rows from table I consisting of the reflections $-\mathrm{g}, 0, \mathbf{g}, 2 \mathrm{~g}$, have been calculated by means of a Bloch wave algorithm without absorption [20]. The relative excitation of the systematic row averaged over specimen thickness $d=50 \mathrm{~nm}$, resp. $d=150 \mathrm{~nm}$ is given in table III. The results of table III clearly indicate that the high order reflections of the systematic row do not influence the total signal from the reflections of the excited 2-beam case. The strongest contribution of a side reflection is obtained in the $(0,2,2)$-case where it ranges around $1 \%$ of the total signal. If the momentum transfer from the 2-beam reflections $(0, \mathrm{~g})$ is $q_{0}=16 \AA^{-1}$ the resulting momentum transfer to the second order reflections $-\mathrm{g}=(0,2,2)$ and $2 \mathrm{~g}=(0,4,4)$ would be $q_{1}=16.7 \AA^{-1}$, which is too close to $q_{0}$ to give an observable change in the shape of the Compton profile of momentum tranfer $q_{0}$.

\section{Raw data.}

The experimental procedure to obtain directional Compton profiles by means of electron energyloss spectrometry in a transmission electron microscope, which can be compared to Compton 
profiles from calculations or other measurements is described in detail elsewhere $[9,10]$. This procedure includes removal of detector caused noise, 'elastic'-background subtraction, deconvolution with respect to plasmon-Compton channel coupling, deconvolution with respect to a finite momentum resolution and normalization of the resulting profile.

\section{Reconstruction of Compton profiles in arbitrary directions.}

Results of photon Compton experiments as well as results of theoretical calculations are usually presented for a few high symmetry directions. To be able to compare such results with the outcome of an electron Compton measurement ${ }^{3}$ it is necesary to have a method to reconstruct Compton profiles for an arbitrary direction from a set of given profiles. This can be achieved by inverting an expansion of the Compton profile in a series of cubic harmonics [13, 14].

Using the fact that the momentum distribution $\rho(\mathbf{p})$ must remain invariant under all operations of the point group of the crystal, $\rho(\mathbf{p})$ may be expanded in lattice harmonics of the proper symmetry; for a cubic crystal these functions are the cubic harmonics

$$
\rho(\mathbf{p})=\sum_{l, d} G_{l, d}(p) K_{l, d}\left(\boldsymbol{\Omega}_{p}\right) .
$$

The direction of $\mathbf{p}$ in the reciprocal lattice of the crystal is given by $\boldsymbol{\Omega}_{p}, d$ distinguishes the various irreducible representations for a specific value of $l$. The cubic harmonic $K_{l, d}\left(\boldsymbol{\Omega}_{p}\right)$ form an orthonormal set of linear combinations of spherical harmonics $Y_{l m}$ of order $l[12]$

$$
\begin{gathered}
K_{l, d}\left(\Omega_{p}\right)=\sum_{m=0,4, . .} b_{l m, d} C_{l m}\left(\Omega_{p}\right) \\
C_{l 0}=Y_{l 0} \\
C_{l m}=\frac{1}{\sqrt{2}}\left(Y_{l m}+Y_{l-m}\right) \quad m \neq 0 .
\end{gathered}
$$

From (4) it is straight forward to show that the Compton profile can also be expanded in a series of cubic harmonics $[13,2]$

$$
\begin{array}{r}
J_{h k l}\left(p_{z}\right)=2 \pi \sum_{l, d} g_{l, d}\left(p_{z}\right) K_{l, d}(h k l) \\
g_{l, d}\left(p_{z}\right)=\int_{p_{z}}^{\infty} G_{l, d}(p) P_{l}\left(\frac{p_{z}}{p}\right) p \mathrm{~d} p
\end{array}
$$

$\langle h k l\rangle$ denotes the direction $\Omega_{p}$ in reciprocal space. Due to the cubic symmetry only harmonics with $l=0,4,6,8,10, \ldots$ are present, the others being zero.

In general we have only a limited number of Compton profiles available from other measurements or calculations. The expansion (5), however, is formally infinite and we have to truncate after a number of terms $m$ equal to the number of Compton profiles $n$ available, if we aim to solve (5) for the $g_{l, d}$. This truncation will only be justified if the expansion converges rapidly enough. Assuming this is the case, the set of equations (5) can be written in matrix notation as

$$
\mathbf{J}=2 \pi \mathbf{K} \cdot \mathbf{g},
$$

$\left({ }^{3}\right)$ We have already seen that we have no access to the high symmetry directions. 
Table IV. - HF1: Radial functions in the expansion of the momentum density of the valence electrons of silicon in cubic harmonics, from [19].

\begin{tabular}{|c|cccc|c|cccc|}
\hline$p$ (a.u.) & $G_{0,0}$ & $G_{4,1}$ & $G_{6,1}$ & $G_{8,2}$ & $p($ a.u. $)$ & $G_{0,0}$ & $G_{4,1}$ & $G_{6,1}$ & $G_{8,2}$ \\
\hline 0.0 & 6.257 & 0.000 & 0.000 & 0.000 & 1.4 & 0.117 & 0.018 & -0.036 & 0.019 \\
0.2 & 6.230 & -0.012 & -0.007 & -0.002 & 1.6 & 0.048 & 0.003 & -0.014 & 0.006 \\
0.4 & 6.180 & -0.060 & -0.044 & -0.014 & 1.8 & 0.025 & 0.003 & -0.002 & 0.000 \\
0.6 & 5.990 & -0.160 & -0.105 & -0.042 & 2.0 & 0.016 & 0.003 & 0.000 & -0.001 \\
0.8 & 5.283 & -0.256 & -0.029 & -0.133 & 2.4 & 0.011 & 0.001 & 0.000 & 0.000 \\
1.0 & 2.317 & 0.029 & 0.542 & -0.078 & 3.0 & 0.006 & 0.000 & 0.000 & 0.000 \\
1.2 & 0.416 & 0.118 & -0.009 & 0.075 & 4.0 & 0.002 & 0.000 & 0.000 & 0.000 \\
\hline
\end{tabular}

where $\mathbf{K}$ is the matrix with elements $K_{l, d}(h k l)$ and $\mathbf{J}$ and $\mathbf{g}$ are column vectors built up of profiles $J_{h k l}$ and $g_{l, d}$-functions respectively. Inversion yields

$$
\mathbf{g}=(2 \pi)^{-1} \mathbf{K}^{-1} \mathbf{J} .
$$

Having determined the radial expansion coefficients $g_{l, d}\left(p_{z}\right)$ it is straightforward to reconstruct a Compton profile $J_{h k l}\left(p_{z}\right)$ in any arbitrary direction $\langle h k l\rangle$ by means of the expansion (5).

The crucial point in this procedure, of course, is the convergence of the expansion (5). The $l$-th order harmonic is built up from terms of the form $\sin ^{i} \cos ^{j} \phi$, with $i+j=l$; thus, if the expansion is truncated at $l=\lambda$ it will never be able to reproduce angular variations in $J\left(p_{z}\right)$ on a scale $\leq \pi / \lambda$. Unless a very large number of Compton profiles is available, the reconstructed angular variation of the profiles will therefore always contain a residual smearing.

\section{Tables of calculated and measured Compton profiles.}

For a comprehensive discussion of the measured electron Compton profiles, some calculated and experimental Compton profiles from other groups shall be given in the following.

A set of 3 different theoretical calculations have been available in a way so that they could be compared to our measurement. Two of them are based on LCAO models using Hartree-Fock wavefunctions, they are (i) by Seth et al. [19] hereafter denoted HF1 and (ii) by Angonoa et al. [1] hereafter denoted HF2. The third approach is a full potential augmented plane wave FLAPW calculation by Blaas [3].

The results of Seth [19] were given in form of the coefficients $G_{l, d}$ in the expansion of the momentum density of the valence electrons, the first 4 cubic harmonics are listed in table IV. The HF1 Compton profiles have been calculated from the $G_{l, d}$ by means of equation (5).

Angonoa reported total Compton profiles in five directions; HF2 results in directions for the electron Comtpon experiment are calculated following (7) from the values listed in table V.

The FLAPW Compton profile for the valence electrons is given as an expansion in 15 cubic harmonics, of which the first 7 expansion coefficients $g_{l, d}$ are given in table VI. It should be noted that these profiles are not suited for direct comparison with directional CP because no correction for electron correlation effects has been done. The so called Lam-Platzman correction leads to an enhancement of states above the Fermi momentum [11]. Since it is supposed to be isotropic in 
Table V. - HF2: Calculated directional total Compton profiles for silicon and isotropic core contribution (last column), from [1].

\begin{tabular}{|c|cccccc|}
\hline$p_{z}$ (a.u.) & $J_{100}$ & $J_{110}$ & $J_{111}$ & $J_{112}$ & $J_{221}$ & core \\
\hline 0.0 & 4.291 & 4.178 & 4.130 & 4.147 & 4.137 & 1.505 \\
0.2 & 4.154 & 4.054 & 4.020 & 4.040 & 4.033 & 1.500 \\
0.4 & 3.732 & 3.710 & 3.721 & 3.745 & 3.722 & 1.484 \\
0.6 & 3.080 & 3.208 & 3.220 & 3.199 & 3.208 & 1.457 \\
0.8 & 2.386 & 2.502 & 2.534 & 2.496 & 2.536 & 1.42 \\
1.0 & 1.848 & 1.846 & 1.870 & 1.856 & 1.858 & 1.372 \\
1.2 & 1.534 & 1.496 & 1.486 & 1.500 & 1.487 & 1.316 \\
1.4 & 1.359 & 1.339 & 1.328 & 1.340 & 1.337 & 1.252 \\
1.6 & 1.240 & 1.235 & 1.233 & 1.232 & 1.231 & 1.182 \\
1.8 & 1.140 & 1.139 & 1.146 & 1.137 & 1.138 & 1.106 \\
2.0 & 1.049 & 1.047 & 1.054 & 1.047 & 1.048 & 1.029 \\
2.2 & 0.962 & 0.963 & 0.962 & 0.961 & 0.961 & 0.949 \\
2.5 & 0.840 & 0.842 & 0.842 & 0.839 & 0.838 & 0.832 \\
3.0 & 0.656 & 0.655 & 0.655 & 0.657 & 0.657 & 0.623 \\
3.5 & 0.503 & 0.505 & 0.513 & 0.507 & 0.507 & 0.504 \\
4.0 & 0.389 & 0.389 & 0.384 & 0.388 & 0.388 & 0.388 \\
\hline
\end{tabular}

Table VI. - FLAPW: Expansion coefficients $g_{l, d}$ for the Compton profile, from [3].

\begin{tabular}{|c|ccccccc|}
\hline$p_{z}$ [a.u. & $g_{0,0}$ & $g_{4,1}$ & $g_{6,1}$ & $g_{8,2}$ & $g_{10,2}$ & $g_{12,2}$ & $g_{12,3}$ \\
\hline 0.0 & 3.0913 & 0.0034 & -0.0310 & -0.0045 & 0.0019 & -0.0021 & -0.0011 \\
0.2 & 2.9616 & 0.0135 & -0.0106 & 0.0070 & -0.0023 & 0.0014 & 0.0003 \\
0.4 & 2.5741 & 0.0156 & 0.0373 & -0.0006 & 0.0023 & -0.0007 & 0.0018 \\
0.6 & 1.9389 & -0.0137 & 0.0131 & -0.0139 & -0.0028 & -0.0004 & -0.0035 \\
0.8 & 1.0991 & -0.0306 & -0.0475 & 0.0160 & 0.0019 & 0.0046 & 0.0046 \\
1.0 & 0.3630 & 0.0051 & 0.0195 & -0.0043 & -0.0002 & -0.0020 & -0.0008 \\
1.2 & 0.1423 & 0.0044 & 0.0029 & -0.0005 & 0.0003 & 0.0002 & -0.0002 \\
1.4 & 0.0912 & 0.0008 & 0.0002 & 0.0001 & 0.0001 & 0.0001 & 0.0001 \\
1.6 & 0.0696 & -0.0002 & -0.0001 & 0.0003 & -0.0006 & 0.0002 & 0.0000 \\
1.8 & 0.0573 & 0.0002 & 0.0002 & 0.0002 & -0.0006 & 0.0002 & 0.0000 \\
2.0 & 0.0490 & 0.0008 & -0.0001 & 0.0001 & -0.0004 & 0.0002 & 0.0000 \\
2.2 & 0.0426 & 0.0010 & -0.0006 & 0.0000 & 0.0001 & 0.0001 & 0.0000 \\
2.4 & 0.0370 & 0.0010 & -0.0010 & -0.0002 & 0.0005 & -0.0001 & 0.0001 \\
2.6 & 0.0319 & 0.0007 & -0.0007 & -0.0003 & 0.0006 & -0.0002 & 0.0000 \\
2.8 & 0.0276 & 0.0002 & -0.0002 & -0.0003 & 0.0003 & -0.0003 & -0.0001 \\
3.0 & 0.0236 & -0.0003 & 0.0004 & 0.0000 & -0.0001 & -0.0001 & -0.0001 \\
4.0 & 0.0090 & 0.0003 & -0.0004 & -0.0001 & 0.0002 & -00002 & -0.0001 \\
\hline
\end{tabular}


Table VII. - Directional X-ray Compton profiles by [18].

\begin{tabular}{|c|ccc|c|ccc|}
\hline$p_{z}$ [a.u.] & $J_{100}$ & $J_{110}$ & $J_{111}$ & $p_{z}$ [a.u.] & $J_{100}$ & $J_{110}$ & $J_{111}$ \\
\hline 0.0 & 2.81 & 2.83 & 2.75 & 0.9 & 0.75 & 0.79 & 0.84 \\
0.1 & 2.75 & 2.78 & 2.70 & 1.0 & 0.56 & 0.54 & 0.62 \\
0.2 & 2.62 & 2.65 & 2.59 & 1.1 & 0.41 & 0.36 & 0.40 \\
0.3 & 2.47 & 2.47 & 2.46 & 1.2 & 0.29 & 0.25 & 0.24 \\
0.4 & 2.27 & 2.25 & 2.25 & 1.3 & 0.21 & 0.17 & 0.17 \\
0.5 & 2.02 & 1.96 & 1.97 & 1.4 & 0.13 & 0.12 & 0.11 \\
0.6 & 1.66 & 1.64 & 1.69 & 1.5 & 0.09 & 0.08 & 0.06 \\
0.7 & 1.28 & 1.35 & 1.37 & 1.6 & 0.05 & 0.06 & 0.04 \\
0.8 & 0.99 & 1.06 & 1.10 & 1.7 & 0.02 & 0.03 & 0.01 \\
\hline
\end{tabular}

momentum space, the FLAPW calculation yields correct results when calculating the differences in the profiles in the various directions.

Directional CPs in the $\langle 100\rangle,\langle 110\rangle$ and $\langle 111\rangle$ direction have been measured by Schülke using $\mathrm{MoK}_{\alpha}$ radiation [18]. Separation of the core and the valence electron part of the CP has been done by subtracting a calculated $2 s^{2} 2 p^{6}$ core profile which has been fitted to the experimental profile between $1.8 \leq p_{x} \leq 3.0$ a.u.; the resulting valence CPs are listed in table VII. Hereafter we will refer to this results as 'SX'.

A $160 \mathrm{keV} \gamma$-ray study of the Compton profiles of the isostructural elements diamond, silicon and germanium done by Reed et al. provides us with valence electron profiles in the directions $\langle 100\rangle,\langle 110\rangle,\langle 111\rangle,\langle 112\rangle$ and $\langle 221\rangle[15]$. Hereafter we will refer to this results as 'R $\gamma$ '.

\section{Electron Compton Profiles.}

The simulated results concerning the systematic row as well as calculations concerning coherent scattering [10] would indicate that the choice of the particular 2-beam case doesn't influence the outcome of a measurement. But we have observed a yet unexplained but reproducible effect which causes the Compton profiles to broaden on their high energy-loss side in the vicinity of the transition between the valence and the core Compton profile. This effect is most pronounced when a low-indexed Bragg reflection $\langle 1,1,1\rangle$ or $\langle 2,2,0\rangle$ is used for the symmetric two-beam case scattering geometry; for the higher indexed reflections $\langle 4,0,0\rangle$ or $\langle 4,2,2\rangle$ we obtained a broadening of the same kind but quantitatively smaller.

Figure 3a,b show two spectra with different g-vector of the Bragg-excited beam, obtained from the same area of a specimen with foil normal [111] and thickness $D_{p}=0.80$ compared to the respective simulated one; the momentum transfer $q=15.6 \AA^{-1}$ is equal in both measurents; figure $3 \mathrm{c}, \mathrm{d}$ show two spectra from the same area of another [111] specimen with specimen thickness $D_{p}=0.90$, the momentum transfer is $q=15.7 \AA^{-1}\langle 2,2,0\rangle$ and $q=16.9 \AA^{-1}\langle 4,2,2\rangle$ respective. A distinct deviation from the simulation is observed for the scattering profils from the $\langle 2,2,0\rangle$ case, although the conditions of the measurement are the same as for the $\langle 4,2,2\rangle$-case, except that the crystal is orientated in a different direction.

There could be various reasons for such a broadening: 

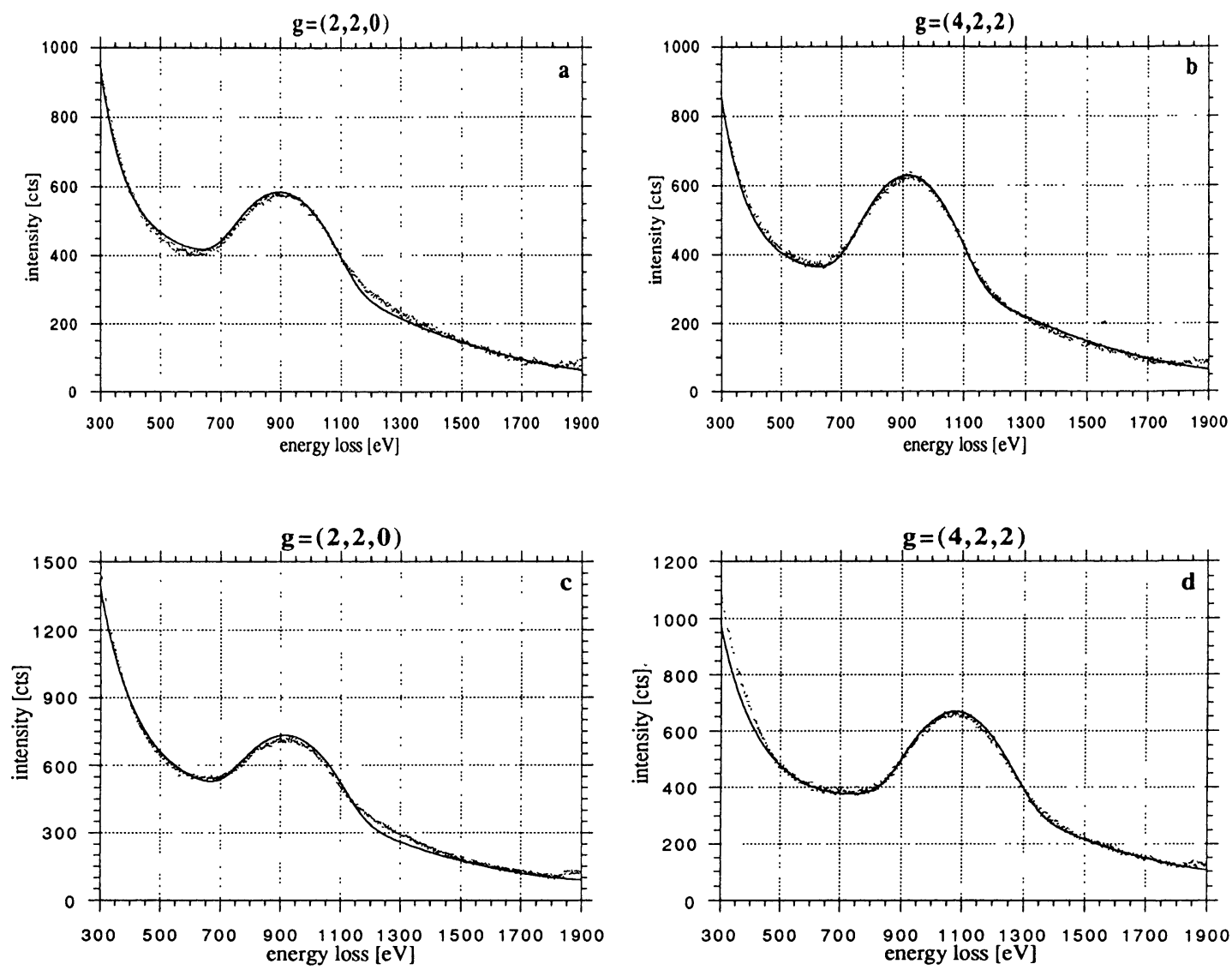

Fig.3. - Total scattering profiles obtained for a $\langle 2,2,0\rangle(\mathrm{a}),\langle 4,2,2\rangle$ (b) 2-beam case from a specimen with $D_{p}=0.80$; total scattering profiles obtained for a $\langle 2,2,0\rangle(\mathrm{c}),\langle 4,2,2\rangle$ (d) 2-beam case from a specimen with $D_{p}=0.90$.

a) Bragg-Compton channel coupling: although the crystal is tilt into a 'two-beam' case indicating that the residual Bragg-reflections are negligible, the sum of the contribution of all Bragg-diffracted beams could give a non negligible contribution to the total spectrum. It has been shown by Williams, for a crystal in a zone-axis orientation, that the contribution of Bragg-Compton channel coupling contributes mainly on the low energy-loss side of the spectrum [24]. This argument holds, qualitatively, also for the case of a tilted crystal, therefore we may expect the major deviation from the simulation in the pre-Compton energy-loss region. Nevertheless we observe from figure 3 that the spectra seem to be not broadend at all on their low energy-loss side.

By tilting the crystal away from the two-beam case into the systematic row, the residual influence of Bragg-Compton channel coupling may be demonstrated experimentally. Figure 4 gives two spectra measured under equal conditions except that the orientation of the crystal has slightly been changed from the two-beam condition (a), to the respective systematic row (b). The intensity of the high energy-loss side remains almost unchanged, indicating that any side reflection does not significantly influence this feature. The low energy-loss side changes 

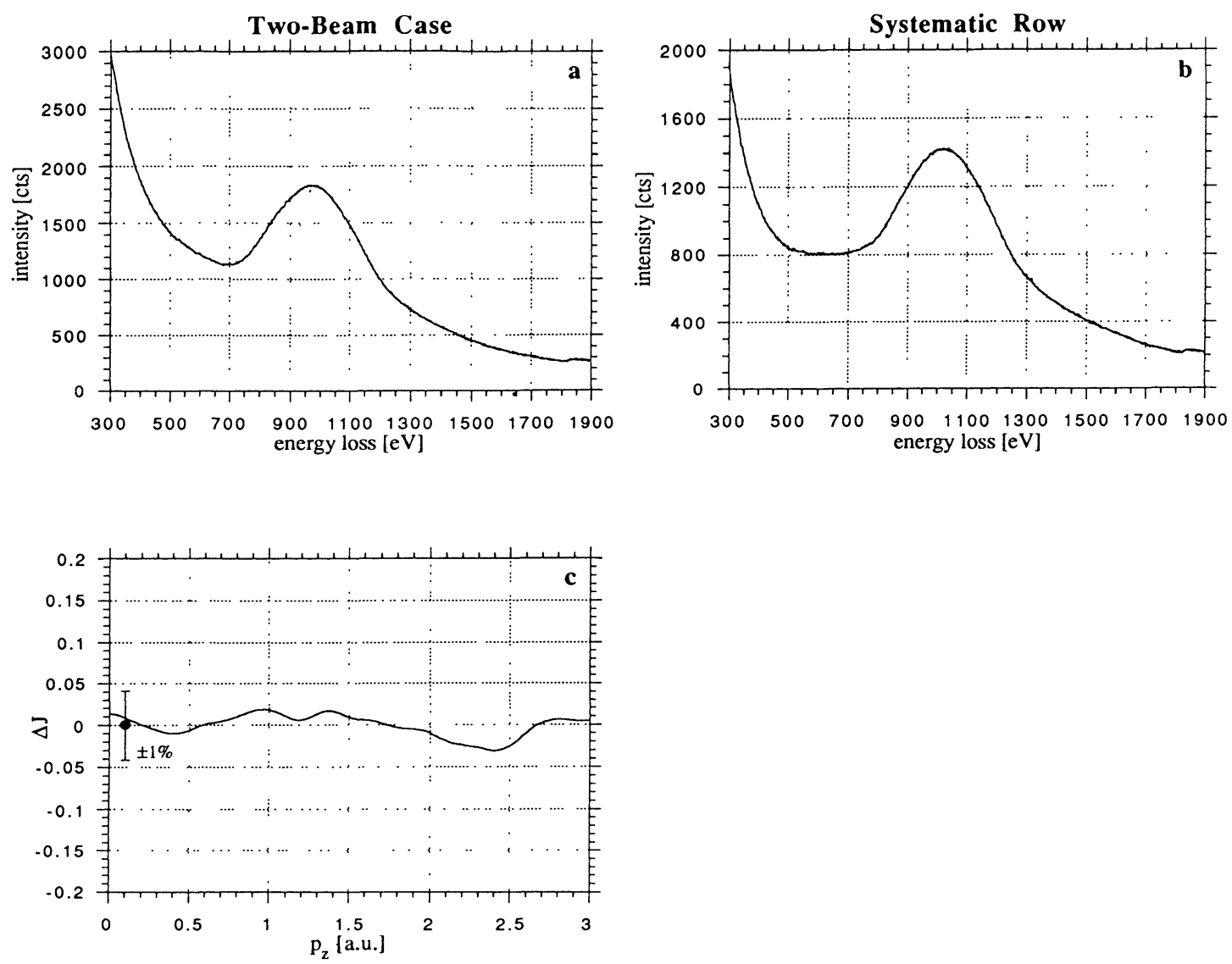

Fig.4. - Total spectra obtained in the $\langle 4,2,2\rangle$ two-beam case (a) and in the $\langle 4,2,2\rangle$ systematic row (b). Difference of the spectra on their high energy-loss side converted to a momentum scale (c).

according probably to the changing excitations strength of the Bragg-reflections in the very vicinity of the spectrometer aperture. Figure $4 \mathrm{c}$ proves that the two profiles, after beeing converted to a momentum scale and normalized, are identical within the statistical limits ${ }^{4}$.

b) The spectra could have been broadend by an additional layer of oxygen on the specimen surface, if the Compton profile of such a contamination is distinctly broader than that of the specimen. But the Compton profiles are supposed to be symmetric with respect to the maximum, thus such a broadening must also be symmetric which is in contradiction to the experimental observations. Below we will give an estimation, proving, by means of microanalytical arguments, that the broadening can not be caused by an oxygen layer upon the crystals surface.

c) If the effective momentum resolution of the measurement is worse than the assumed resolution in the simulation we may also expect a deviation of the experimental profile from the simulated. Nevertheless such a deviation has got to be symmetric with respect to the

$\left({ }^{4}\right)$ The noise in the profiles due to counting statistics is indicated by the error bar; it was determined from the raw data to be $\pm 1 \%$ at $p_{z}=0$. 
Compton profile peak and it can be shown by means of the multiple scattering simulation that the quantity of the deviation can not be explained by a limited momentum resolution.

d) As shown by Su et al. [21] plasmon-Compton channel coupling alters the spectra mainly on their high energy-loss side which would qualitatively confirm the experimental observations. Since the simulated spectra do account for the plasmon-Compton channel coupling it follows that the effects of plasmon-channel coupling upon the profile are far below the observed broadening effect. Such a strong deviation from simulated profiles could only be explained by a grossly wrong assumption of the cross section for plasmon excitation in the simulation; this is very unlikely.

In the following we will discuss some measured electron Compton profiles obtained in a highindexed two-beam scattering geometry $(\langle 4,0,0\rangle,\langle 4,2,2\rangle)$. First we will compare the measured direct profiles $J_{\exp }\left(p_{z}\right)$ to theoretical HF2-profiles $J_{\text {th }}\left(p_{z}\right)$ on a momentum scale which refers to $p_{z}$. Figure 5 shows several difference profiles $\Delta J=J_{\mathrm{th}}-J_{\exp }$ between the measured electron Compton profiles in the $\langle 19,3,2\rangle,\langle 22,19,4\rangle$ and $\langle 9,5,4\rangle$-direction in momentum space and the respective theoretical HF2-profiles.

Some direction dependent deviations from the theoretical profile indicate a disagreement of the measurement with the calculation due to possible insufficiencies of the theoretical model in use. But as already discussed we observe a second kind of deviation for all profiles at momentum values around $p_{z}=1 \mathrm{a}$.u. that kind that the experimental profile is too broad in the transfer region between the valence electron profile and the core electron profile. This deviation is not reproducible in its quantity and it seems not to be dependend on the crystal orientation in a systematic manner; therefore we cannot ascribe this effect any relevance concerning the physical properties of the sample to be measured.

The same picture but even worse arises when we compare the measured profiles $J_{\exp }\left(p_{z}\right)$ with those obtained by the $\gamma$-ray measurements $J_{\mathrm{R}_{\gamma}}\left(p_{z}\right)$ which have been reconstructed from table VIII. Figure 6 displays the differences $\Delta J=J_{\mathrm{R}_{\gamma}}-J_{\mathrm{el}}$ for two different directions. A deviation of equivalent kind as discussed above located at the same momentum value but even more pronounced and sharper can be observed.

This broadening of the measured valence electron profile can not be explaind by means of the multiple scattering calculations nor by any resolution effects ${ }^{5}$. The possibility remains that they are caused by thin layers of oxide on the sample surface. A short simulation discusses this question.

For this purpose we construct an artificial $\mathrm{SiO}_{2}$ profile by taking an oxygen $1 s^{2} 2 s^{2}$-core plus a silicon $1 s^{2} 2 s^{2} 2 p^{6}$-core and a Gaußian shaped valence profile of 1.8 a.u half-width; the profile is normalized to have an area of 30 the number of electrons in the $\mathrm{SiO}_{2}$-particle. This $\mathrm{SiO}_{2}$ profile is added to a silicon HF2-profile in varying relations $\mathrm{Si}: \mathrm{SiO}_{2}$ and the resulting 'contaminated' $\mathrm{Si}$ profile is then normalized to 14 the number of electrons in the pure $\mathrm{Si}$. Figure $7 \mathrm{~b}$ shows the deviation of the 'contaminated' profile from the pure profil for relations $\mathrm{Si}_{\mathrm{SiO}}=10: 1,20: 1$, $50: 1$, compared to the deviation of a measured profile in the $\langle 19,3,2\rangle$ direction from the respective HF2 profile. Following figure 7 the amount of the deviation could be explained with the presence of $\mathrm{SiO}_{2}$ in relation to pure $\mathrm{Si}$ of $\mathrm{Si}: \mathrm{SiO}_{2}=10: 1$.

Such an enormous amount of oxygen on the $\mathrm{Si}$ is detectable with EELS microanalysis. Figure 8 compares calculated bright-field high energy-loss spectra in the region of the $\mathrm{O}_{2} \mathrm{~K}$-edge with the respective measured spectrum; the theoretical spectra have been calculated following the hydrogenic approach by Egerton [4]. The measurement yields no detectable amount of oxygen within the Si background in the limits of the counting statistics which are below the level of the $\mathrm{Si}: \mathrm{SiO}_{2}=50: 1$ signal. This means that the variances of the measured electron Compton profiles

$(5)$ Which we claim to remove by a deconvolution procedure $[21,10]$. 

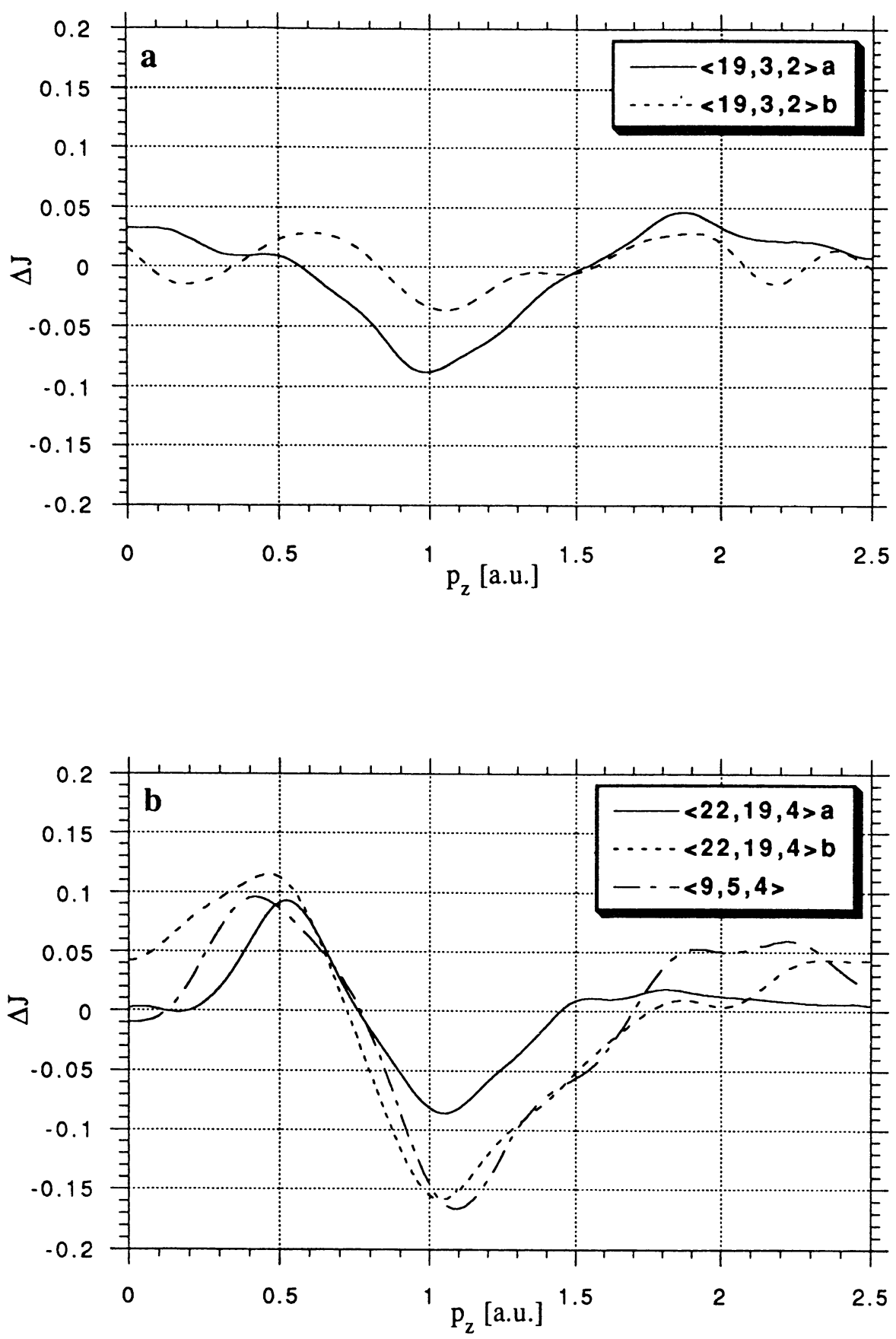

Fig.5. - Difference profile $\Delta J=J_{\mathrm{HF} 2}-J_{\mathrm{exp}}$ between the profiles of the theory HF2 and experimental pro-

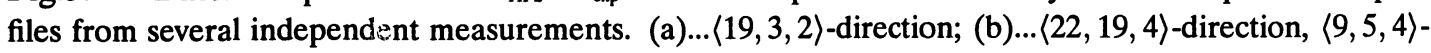
direction. 

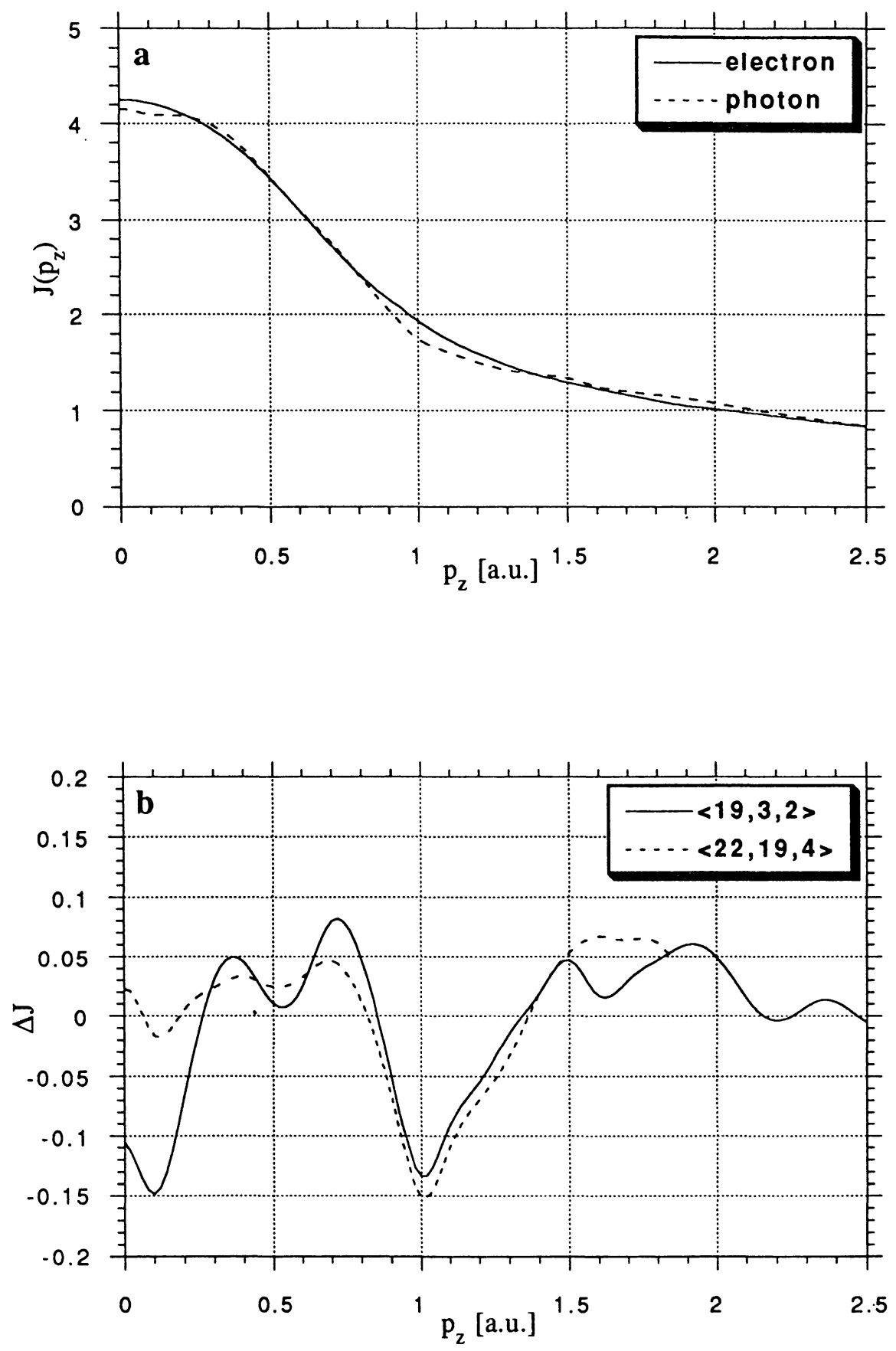

Fig.6. - (a) Direct comparsion of the Compton profile in $\langle 19,3,2\rangle$ direction measured by means of electron scattering and $\gamma$-ray scattering. (b)...Difference profile $\Delta J=J_{\mathrm{R}_{\gamma}}-J_{\text {exp }}$ between the $\gamma$-ray profile $J_{\mathrm{R} \gamma}$ and the electron scattering profile $J_{\exp }$ for $\langle 19,3,2\rangle$ and $\langle 22,19,4\rangle$. 

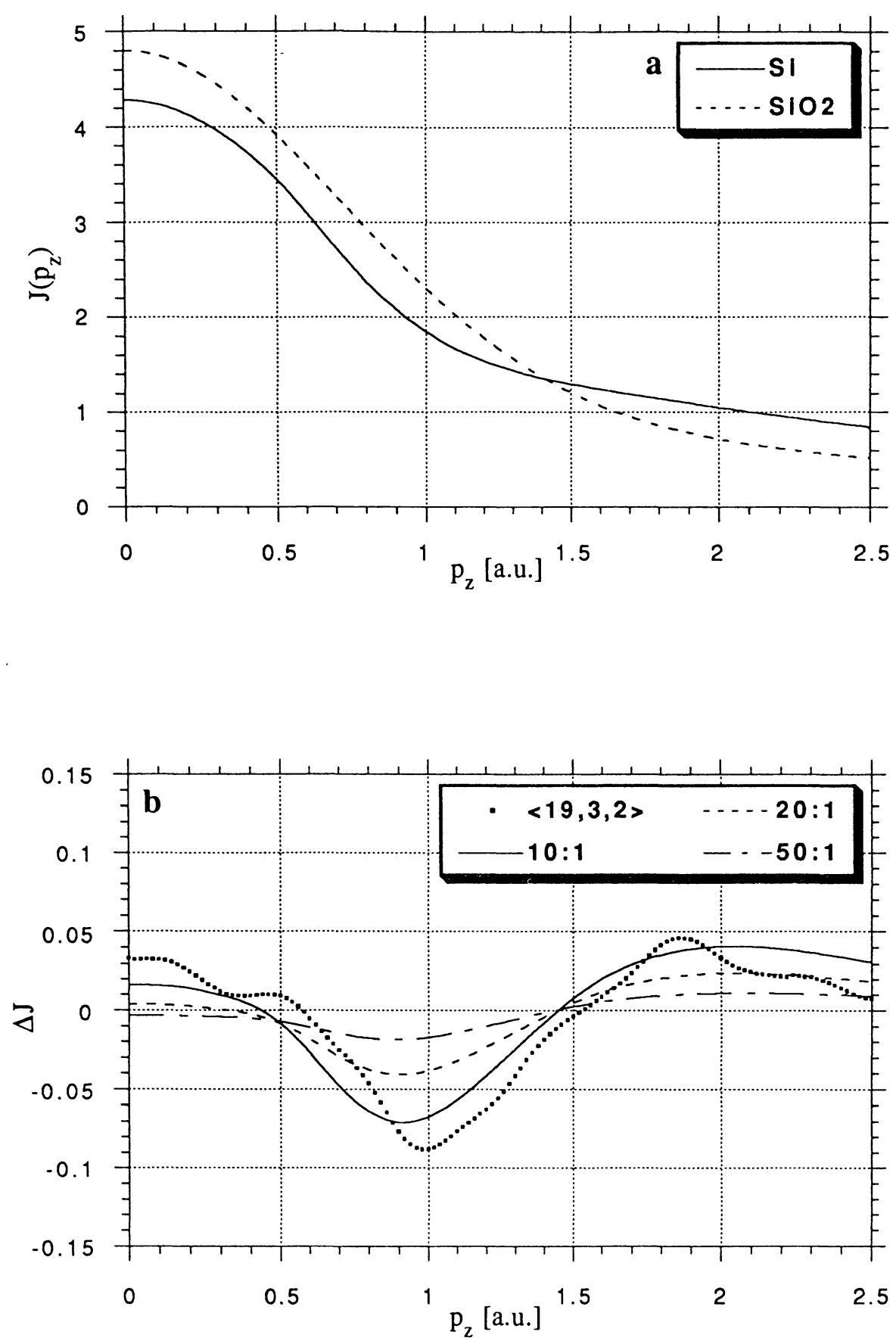

Fig.7. - (a) $\mathrm{Si}$ and artificial $\mathrm{SiO}_{2}$ Compton profile; the $\mathrm{SiO}_{2}$ profile has been normalized to have the same area as the Si profile. (b)...Deviations of the 'contaminated' profile from the pure Compton profile for various relations of $\mathrm{Si}_{\mathrm{SiO}}$, compared to a measured variance $\Delta J=J_{\mathrm{HF}}-J_{\exp }$ in $\langle 19,3,2\rangle$ direction. 
Table VIII. - R $\gamma: \gamma$-ray directional Compton profiles and $1 s^{2} 2 s^{2} 2 p^{6}$ core, from [15].

\begin{tabular}{|c|c|c|c|c|c|c|}
\hline$p_{z}[$ a.u. $]$ & $J_{100}$ & $J_{110}$ & $J_{111}$ & $J_{112}$ & $J_{221}$ & core \\
\hline 0.0 & 2.569 & 2.622 & 2.548 & 2.593 & 2.579 & 1.569 \\
\hline 0.1 & 2.562 & 2.546 & 2.531 & 2.537 & 2.558 & 1.567 \\
\hline 0.2 & 2.543 & 2.482 & 2.488 & 2.479 & 2.488 & 1.562 \\
\hline 0.3 & 2.444 & 2.338 & 2.411 & 2.402 & 2.377 & 1.554 \\
\hline 0.4 & 2.237 & 2.143 & 2.225 & 2.213 & 2.192 & 1.542 \\
\hline 0.5 & 1.943 & 1.888 & 1.952 & 1.931 & 1.940 & 1.527 \\
\hline 0.6 & 1.614 & 1.629 & 1.659 & 1.632 & 1.656 & 1.509 \\
\hline 0.7 & 1.278 & 1.363 & 1.339 & 1.333 & 1.339 & 1.487 \\
\hline 0.8 & 0.928 & 1.028 & 0.980 & 0.994 & 0.992 & 1.462 \\
\hline 0.9 & 0.604 & 0.674 & 0.631 & 0.634 & 0.643 & 1.434 \\
\hline 1.0 & 0.347 & 0.368 & 0.365 & 0.347 & 0.359 & 1.403 \\
\hline 1.1 & 0.245 & 0.233 & 0.248 & 0.239 & 0.239 & 1.369 \\
\hline 1.2 & 0.175 & 0.148 & 0.148 & 0.154 & 0.151 & 1.333 \\
\hline 1.3 & 0.138 & 0.105 & 0.105 & 0.111 & 0.108 & 1.295 \\
\hline 1.4 & 0.119 & 0.101 & 0.113 & 0.104 & 0.101 & 1.255 \\
\hline 1.5 & 0.101 & 0.101 & 0.116 & 0.116 & 0.092 & 1.214 \\
\hline 1.6 & 0.085 & 0.106 & 0.097 & 0.106 & 0.109 & 1.171 \\
\hline 1.7 & 0.077 & 0.101 & 0.086 & 0.077 & 0.089 & 1.128 \\
\hline 1.8 & 0.076 & 0.091 & 0.073 & 0.064 & 0.070 & 1.084 \\
\hline 1.9 & 0.079 & 0.079 & 0.070 & 0.073 & 0.067 & 1.040 \\
\hline
\end{tabular}

from theory and the results of the photon experiments can not be explained with the presence of an oxygen layer. So we have to conclude that there is no answer to the question of the varying quality of the measured Compton profiles at this time, it was not possible to observe any kind of reproducability concerning the broadening of the profiles.

Since systematic errors are supposed to vanish when calculating differential profiles, we may expect to observe differential profiles which are closer to the theory, provided that the deviations described above are of the same quantity in both profiles which are to be subtracted. Figure 9 shows differential profiles between the directions $\langle 19,3,2\rangle$ and $\langle 22,19,4\rangle$ obtained in two independent measurements compared to those of the HF2-theory (Fig. 9a), respective to the HF1and FLAPW-theory (Fig. 9b). The measurements are reproducible concerning the main features, but show slight quantitative variations due to the varying influence of the broadening as described above.

Nevertheless we are able to state that the measurements support the predictions of the HF2 calculations rather than HF1 and FLAPW, which predict a zero crossing of the differential profile 


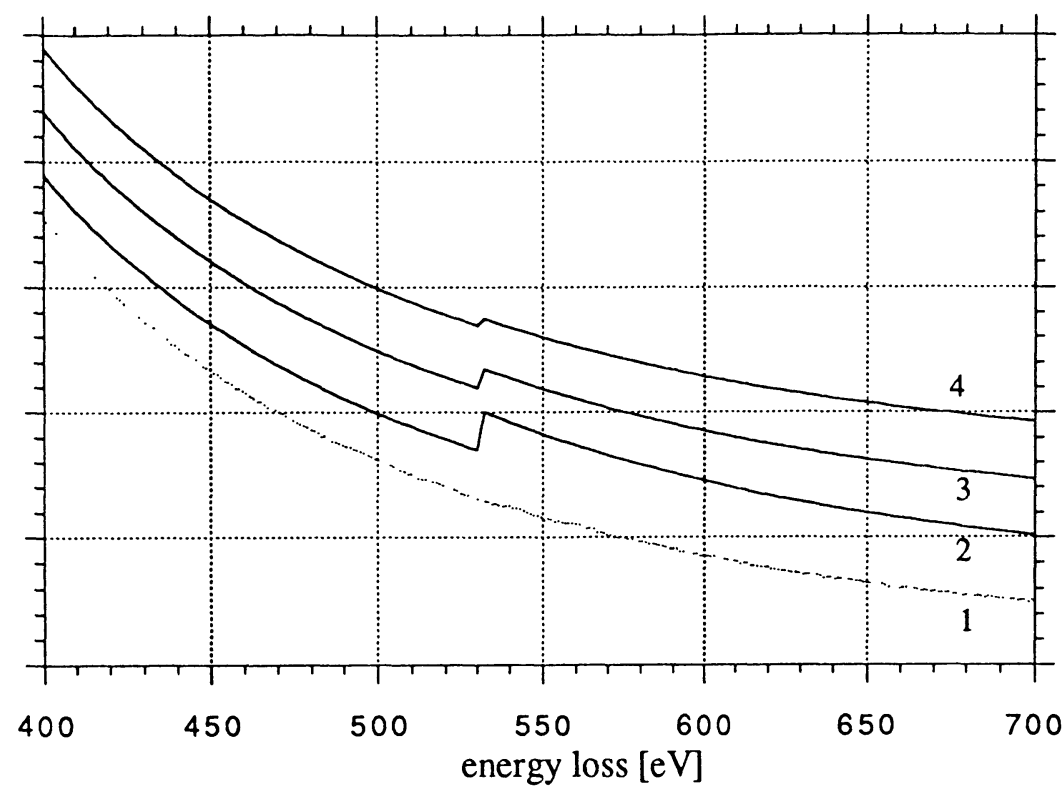

Fig.8. - Measured (1) and calculated bright-field high-loss spectra; $\mathrm{Si}_{\mathrm{SiO}}=10: 1$ (2), $\mathrm{Si}_{2} \mathrm{SiO}_{2}=20: 1$ (3), $\mathrm{Si}: \mathrm{SiO}_{2}=50: 1(4)$.

around $p_{z}=0.15$ a.u.. The electron Compton measurements yield no evidence for such a zero crossing. Figure 10 compares the electron scattering results with those of the reconstructed $\gamma$-ray and X-ray values; we observe that the photon measurements are more likely the HF1 and FLAPW calculations since they exhibit a zero crossing in the region $p_{z}<0.4$ a.u..

The direction in momentum space which has been achieved with the measurement in the $(4,2,2)$ case near the [011] zone axis, tilting the specimen for an angle of $\phi=11^{\circ}$ and selecting a scattering angle of $q=15.2 \AA^{-1}$, is $\langle 9,5,4\rangle$. The angle $\beta$ to the direction $\langle 22,19,4\rangle$ measured near the $[1,1,1]$ pole is $\beta=17.6^{\circ}$. The calculations yield an interesting picture since they all predict qualitatively the same shape for the difference profile $\Delta J=J_{9,5,4}-J_{22,19,4}$, but quantitatively that by HF 2 is almost an order of magnitude smaller than those by the HF1 and the FLAPW calculation. The results of the electron measurements indicate no anisotropy at all within the statistical limits, see figure 11a. Figure 11b shows that the photon experiments, particulary that by Reed [15], have been able to measure an anisotropy qualitatively equal to the theoretical predictions. Therefore we may conclude that it seems that the electron experiments are less sensitiv than the photon experiments.

\section{Conclusions.}

A transmission electron microscope equipped with a parallel electron energy-loss spectrometer is under particular conditions suited for the measurement of momentum density distributions of charge carriers in a solid on a scale of $\mu \mathrm{m}$. At present the momentum resolution which can be achieved in the TEM is comparable to modern synchrotron experiments using bent crystal spectrometers. This resolution could be enhanced at the expense of the count rate, demanding either an electron source of higher effectivity (field emission gun) or a detection system which is capable of single electron detection. 

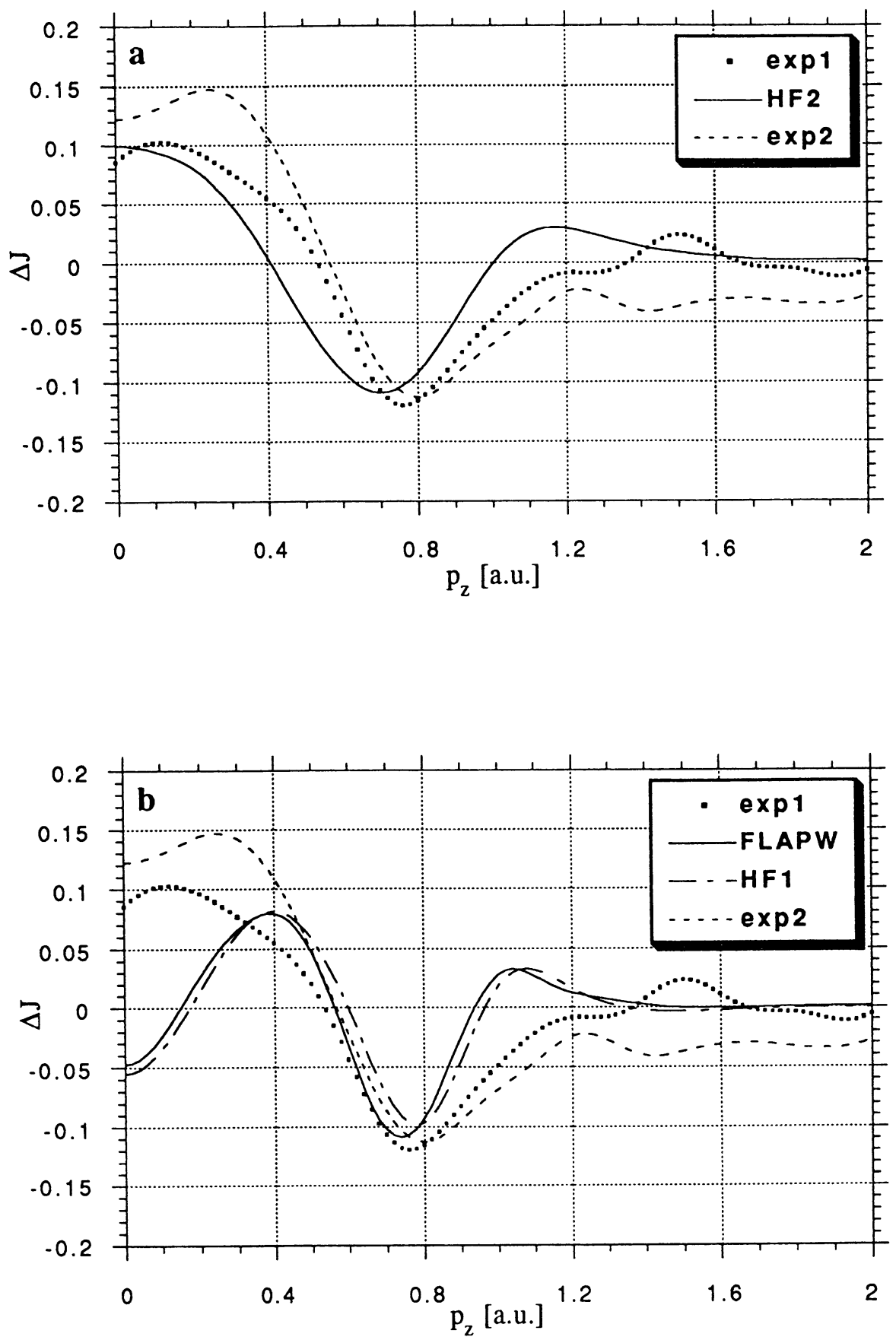

Fig.9. - (a) Measured anisotropy in the Compton profile $\Delta J=J_{19,3,2}-J_{22,19,4}$ compared to the respective HF2 result. (b)...Dito compared to the FLAPW, resp. HF1 theory. 

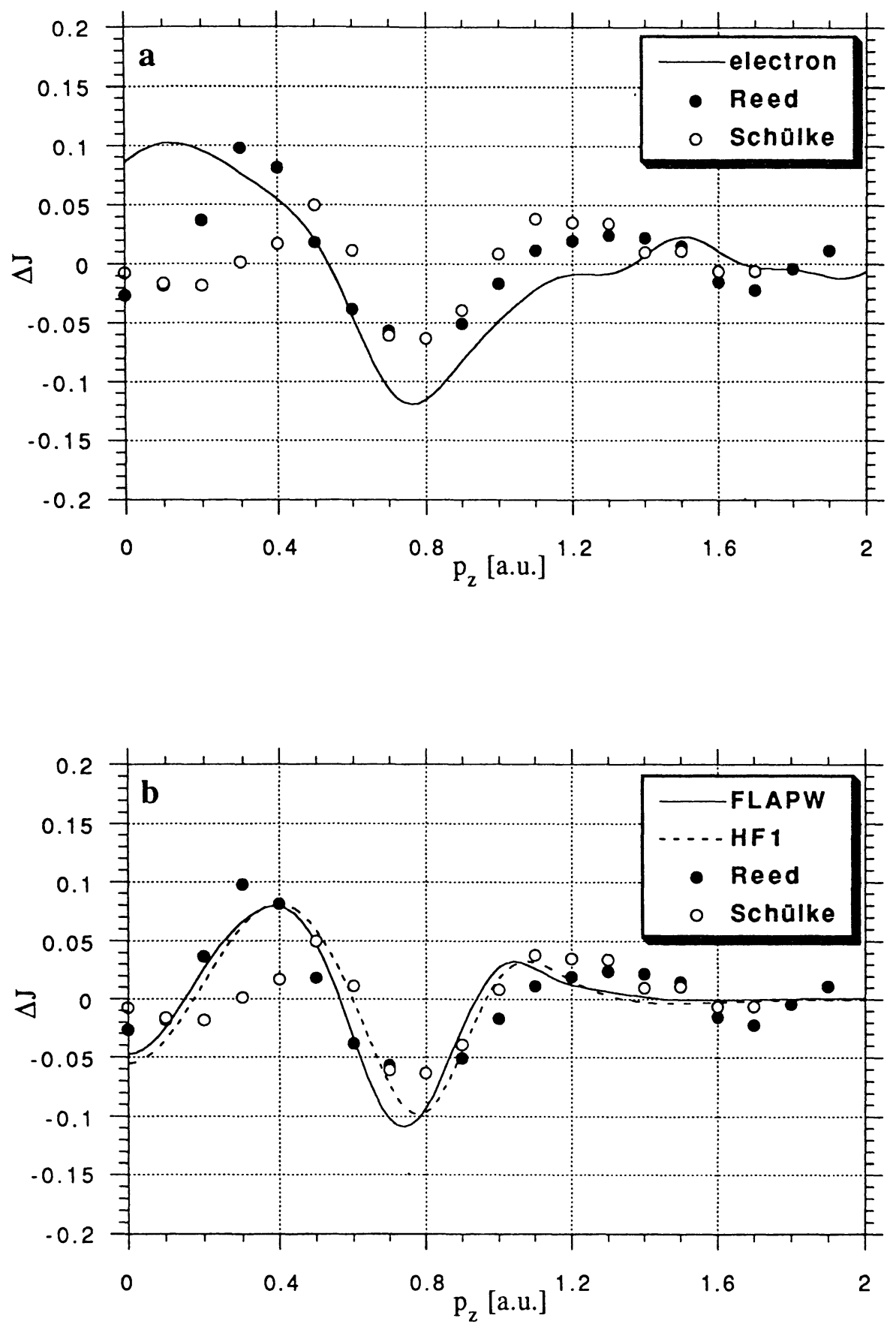

Fig.10. - (a)...Comparison of measured anisotropies in the Compton profile $\Delta J=J_{19,3,2}-J_{22,19,4}$ obtained by means of electron scattering and $\gamma$-ray (Reed), respectively X-ray (Schülke) scattering. (b)...Photon results compared to the HF1 and FLAPW theory. 

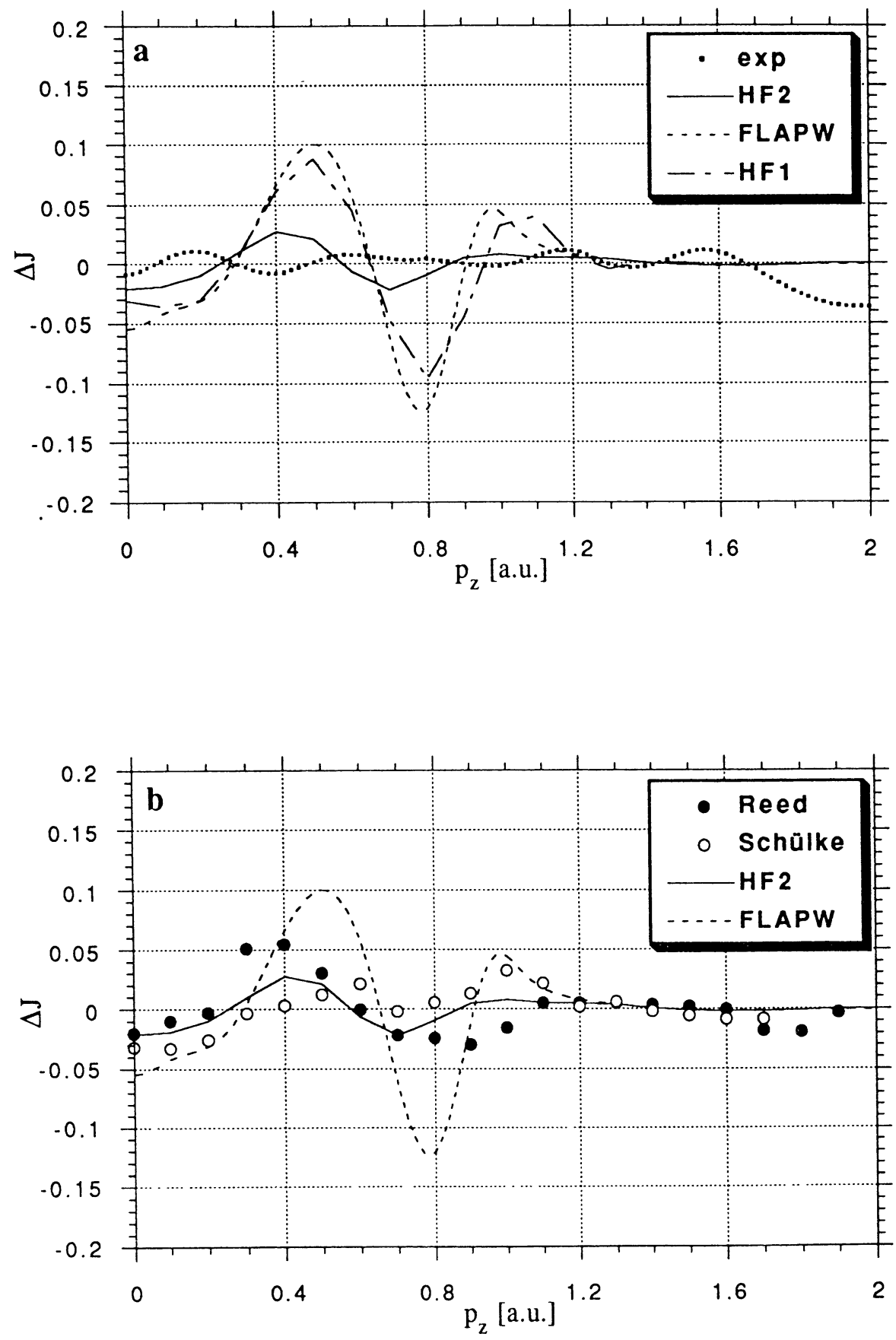

Fig.11. - (a)...Anisotropy $\Delta J=J_{9,5,4}-J_{22,19,4}$ obtained by electron scattering compared to the several calculations. (b) Dito for the photon scattering experiments. 
Specimen support and preparation is one of the crucial points for ECOSS in order to be useful. Attempts to measure the Compton profile of aluminum which easily oxidizes, showed that the Compton spectrum is most sensitive to the presence of an additional oxygen layer on the sample surface.

With a set of single crystal silicon spamples the ability of ECOSS to measure directional anisotropies in the Compton profile was tested. The introduction of a scattering geometry taking advantage of crystal symmetry enabled us to avoid Bragg-Compton channel coupling and to measure Compton profiles of well defined orientation in momentum space. A disadvantage of the symmetric two-beam case setup is that the accesible directions do not coincide with the high symmetry directions usually documented in Compton literature and are not that seperated as the respective high symmetry directions.

With the help of recently developped procedures concerning background subtraction and deconvolution it was possible to measure directional Compton profiles in three different directions from single crystal specimens of three different orientations. Measured anisotropies were in overall qualitative agreement with the theory and previous photon scattering results, but showed differences in some details compared to the photon experiments. The analysis of direct profiles as compared to the respective theoretical and photon experiments showed a strong deviation in the vicinity of the Fermi momentum $q_{\mathrm{F}}$ which could not been explained by means of the multiple scattering calculations or by any specimen contamination.

This broadening effect occurs strongest when using a low-indexed Bragg-reflection like $\langle 1,1,1\rangle$ or $\langle 2,2,0\rangle$ for the symmetric two-beam case scattering geometry, for a higher indexed Braggreflection $(\langle 4,0,0\rangle$ or higher) the profiles are less altered, though the deviation from theoretical and photon results are still obvious. The broadening effect is not reproducible in its quantity and no systematic dependence on any specimen parameter like thickness or orientation was observable. We have no explanation of this effect.

Further experiments with homogeneous, polycrystalline thin films may help answering the question whether there is an intrinsic difference in the results of a Compton measurement when using electrons as probing particles instead of photons.

\section{Acknowledgements.}

This work was supported by the Austrian Fonds zur Förderung der Wissenschaftlichen Forschung, Project P7432-PHY.

\section{References}

[1] Angonoa G., Dovesi R., Pisani C. and Roetti C., Phil. Mag. B44 (1981) 413.

[2] Blaas C., Berechnung von Compton-Profilen über eine Multipolentwicklung der Impulsdichte diploma thesis at (Technische Universität Wien, 1991).

[3] Blaas C., private communication.

[4] Egerton R.F., Electron Energy-Loss Spectroscopy in the Electron Microscope (New York, Plenum, 1986)

[5] Eisenberger P. and Platzman P.M., Phys. Rev. A2 (1970) 415.

[6] Hahn T., ed., International Tables for Crystallography (D. Reidel Publishing Company, 1987).

[7] Hirsch P., Howie A., Nicholson R.B., Pashley D.W. and Whelan M.J., Electron Microscopy of thin Crystals (Robert E. Krieger Publishing Co., Inc. New York, 1965). 
[8] Jonas P. and Schattschneider P., Ultramicroscopy 38 (1991) 117.

[9] P. Jonas and P. Schattschneider, accepted by J. Phys. C (1993)

[10] Jonas P. Electron Compton Scattering in the Electron Microscope, doctoral thesis at (Technische Universität Wien, 1993).

[11] Lam L. and Platzman P.M., Phys. Rev. B9 (1974) 5122.

[12] Mueller F.M. and Priestly M.G., Phys. Rev. 148 (1966) 638.

[13] Mijnarends P.E., Phys. Rev. 160 (1967) 512.

[14] Mijnarends P.E., Chapt. 10 in Compton Scattering, Ed. B.G. Williams (McGraw-Hill, New-York, 1977).

[15] Reed W.A. and Eisenberger P., Phys. Rev. B6 (1972) 4596.

[16] Schattschneider P., Jonas, P. and Mändl M., Microsc. Microanal. Microstruct. 2 (1991) 367.

[17] Schattschneider P. and Jonas P., Ultramicroscopy 49 (1993) 179.

[18] Schülke W. and Lucht W., Phys. Stat. Sol. B59 (1973) K107.

[19] Seth A. and Ellis D.E., J. Phys. C10 (1977) 181.

[20] Stadelmann P.A., Ultramicroscopy 21 (1987) 131.

[21] Su D.S., Jonas P. and Schattschneider P., Phil. Mag. B66 (1992) 405.

[22] Williams B.G., Parkinson G.M., Eckhardt C.J., Thomas J.M. and Sparrow T., Chem. Phys. Lett. 78 (1981) 434.

[23] Williams B.G., Sparrow T.G. and Egerton R.F., Proc. R. Soc. Lond. A393 (1984) 409.

[24] Williams B.G., Uppal M.K. and Brydson R.D., Proc. R. Soc. Lond. A409 (1987) 161. 